\title{
Image Denoising using Combination of Median Filtering and Wavelet Transform
}

\author{
Pankaj Rakheja \\ Assistant Professor \\ Northcap University, Gurgaon
}

\author{
Rekha Vig, PhD \\ Associate Professor \\ Northcap University, Gurgaon
}

\begin{abstract}
Image processing is basically carried out to enhance or restore a noisy image. The former mechanism is known as image enhancement and the later one is known as image restoration. Image gets corrupted with noise during acquisition phase or during transmission phase. Denoising can be done by numerous methods like neighbourhood operations, arithmetic operations, Transforms etc. In this paper we have combined neighbourhood processing techniques with Transform specifically wavelet Transform. The results obtained after simulation show that the combined algorithm performs better than both individually. Simulated results are for Gaussian, Speckle and Salt \& Pepper noise, for denoising median filter of size $3 \mathrm{X} 3,5 \mathrm{X} 5$ and discrete wavelet Transform are used here. Then results obtained were evaluated on the basis of Peak signal to noise ratio which has improved remarkably.
\end{abstract}

\section{General Terms}

Image denoising, Median Filtering, Wavelet Transform

\section{Keywords}

Artifacts, Decomposition, Discrete wavelet transform, Median filter

\section{INTRODUCTION}

Image Restoration is process where we take a corrupt/noisy image and estimate the noise free and original image from it. Image might get corrupted from motion blur, noise and misfocused. It is performed by reversing the process that corrupted the image. This is done by imaging a point source and using the point source image, which is known as the Point Spread Function (PSF) to restore the information in the image which was lost in the blurring process.

Image denoising [2-5] plays a vital role in a wide range of applications such as image registration, image segmentation, and image classification and visual tracking. The image may get degraded by noise from any source during acquisition or transferring through any media that is image may get corrupted from intrinsic or extrinsic source.

Noise Models in Digital Image Processing

Noise is of various types and different types of noises are added to images during various processes as mentioned. The most common types of noise models [1] are:

- Gaussian Noise Model: Gaussian noise is caused by natural sources such as thermal vibration of atoms and discrete nature of radiation of warm objects.

- White Noise: White noise is that whose power spectrum is constant; autocorrelation is zero; it normally disturbs the image pixel values positively.

- Brownian Noise (Fractal Noise): The noise which has its power spectral density proportional to the square of frequency over an octave is known as Brownian noise. It is generated from random movement of suspended particles in fluid or due to white noise.

- Impulse Valued Noise (Salt and Pepper Noise): In this form of noise some pixel values are changed in the image. Image pixel values are replaced by corrupted pixel values either maximum 'or' minimum pixel value that is why it is known as Impulse valued noise. It is mainly caused by malfunctioning of pixel elements in camera sensors, faulty memory space in storage, errors in digitization process and many more

- Periodic Noise: This type of noise is generated from interferences from electronic source, especially in power signal during image acquisition.

- Quantization noise: Quantization noise creeps in amplitude quantization process during analog to digital conversion.

- Speckle Noise: This noise is multiplicative noise. There are found in coherent imaging systems such as laser, radar and acoustics etc,.. Its probability density function follows gamma distribution function

- Photon Noise (Poisson Noise): This type of noise creeps in due to the statistical nature of electromagnetic waves such as $\mathrm{X}$-rays, visible lights and gamma rays. There is random fluctuation of photons in their sources. As a result gathered image has spatial and temporal randomness

- Structured Noise: This type of noise is periodic, stationary or non-stationary and aperiodic in nature. If this noise is stationary, it has fixed amplitude, frequency and phase; Structured noise caused by interferences among electronic components

- Gamma Noise: The laser based images have this sort of noise. Its probability distribution obeys the Gamma distribution.

- Rayleigh noise: Rayleigh noise appears in radar range images

Techniques to remove noise

Spatial filtering [7]

It is method of choice in situations where only additive noise is present in the image. It includes

1) Mean filters

$\mathrm{S}_{\mathrm{xy}}$ represent the set of coordinates in rectangular subimage window of size $\mathrm{mXn}$, centred at point 
$(\mathrm{x}, \mathrm{y}) \cdot \mathrm{g}(\mathrm{x}, \mathrm{y})$ is corrupted image and $\mathrm{f}(\mathrm{x}, \mathrm{y})$ is restored image.

- $\quad$ Arithmetic filter

$$
\mathrm{f}(\mathrm{x}, \mathrm{y})=\frac{1}{\mathrm{mn}} \sum_{(\mathrm{s}, \mathrm{t}) \in \mathrm{S} x \mathrm{~g}}^{\mathrm{g}(\mathrm{s}, \mathrm{t})}
$$

it smooths local variations in image and reduces noise as a result of bluring.

- $\quad$ Geametric mean filter

$$
f(x, y)=\left(\prod_{(s, t) \in S x y} g(s, t)\right.
$$

Smoothens image and looses less information

- Harmonic mean filter

$$
f(x, y)=\frac{m n}{\sum_{(s, t) € S x y} 1 / g(s, t)}
$$

It works well for salt and gaussian noise, but fails for pepper noise

- Contraharmonic mean filter

$$
\mathrm{f}(\mathrm{x}, \mathrm{y})=\frac{\sum_{(\mathrm{s}, \mathrm{t}) \in \mathrm{Sxy}} \mathrm{g}(\mathrm{s}, \mathrm{t})^{\mathrm{Q}+1}}{\sum_{(\mathrm{s}, \mathrm{t}) \in \mathrm{S} x \mathrm{~g}} \mathrm{~g}(\mathrm{~s}, \mathrm{t})^{\mathrm{Q}}}
$$

It works well in reducing salt and pepper noise individually but not both at same time.

2) Order statistic filters

- Median filter

$$
\mathrm{f}(\mathrm{x}, \mathrm{y})=\underset{(\mathrm{s}, \mathrm{t}) € \text { Sxy }}{\operatorname{median}}\{\mathrm{g}(\mathrm{s}, \mathrm{t})\}
$$

It reduces salt and pepper noise effectively

- Max min filter

Max filter reduces pepper noise and Min filter reduces salt noise.

$$
\begin{aligned}
& \mathrm{f}(\mathrm{x}, \mathrm{y})=\max _{(\mathrm{s}, \mathrm{t}) \in \operatorname{Sxy}}\{\mathrm{g}(\mathrm{s}, \mathrm{t})\} \\
& \mathrm{f}(\mathrm{x}, \mathrm{y})= \\
& \min _{(\mathrm{s}, \mathrm{t}) \in \operatorname{Sxy}}\{\mathrm{g}(\mathrm{s}, \mathrm{t})\}
\end{aligned}
$$

- $\quad$ Midpoint filter

$$
\mathrm{f}(\mathrm{x}, \mathrm{y})=0.5 \mathrm{X}\left[\max _{(\mathrm{s}, \mathrm{t}) \in \operatorname{Sxy}}\{\mathrm{g}(\mathrm{s}, \mathrm{t})\}+\underset{(\mathrm{s}, \mathrm{t}) € \operatorname{Sxy}}{\min }\{\mathrm{g}(\mathrm{s}, \mathrm{t})\}\right]
$$

It works well for Gaussian and uniform noise

- Alpha trimmed mean filter

$$
\mathrm{f}(\mathrm{x}, \mathrm{y})=\frac{1}{\mathrm{mn}-\mathrm{d}} \sum_{(\mathrm{s}, \mathrm{t}) \in S x y} \mathrm{~g}_{\mathrm{r}}(\mathrm{s}, \mathrm{t})
$$

Here we delete $d / 2$ lowest and $d / 2$ highest intensity values of $\mathrm{g}(\mathrm{s}, \mathrm{t})$ and $\mathrm{g}_{\mathrm{r}}(\mathrm{s}, \mathrm{t})$ represent remaining $(\mathrm{mn}-\mathrm{d})$ pixels. It works well for Gaussian and Impulse noise.

\section{OVERVIEW}

All Discrete wavelet transform [5, 8-9] is used to find the approximation and detailed coefficients of a discrete signal. It basically represents the time frequency analysis of discrete signal. It gives information about spectral content of the signal at particular location. In wavelet decomposition, signal breaks it into two classes; low pass and high pass. These classes independently carry information of original signal. Figure 1 and 2 below show the DWT decomposition and reconstruction steps of a 2D image signal for level of 2 ;

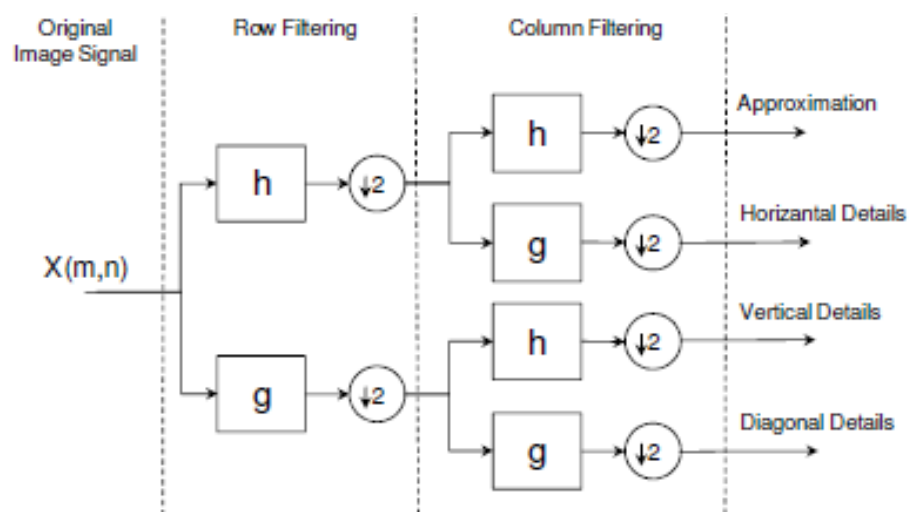

Figure 1: DWT decomposition

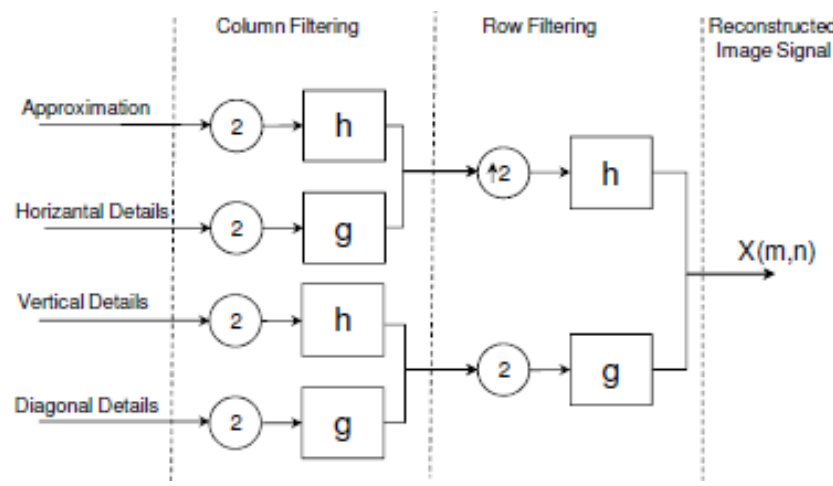

Figure 2: DWT Reconstruction

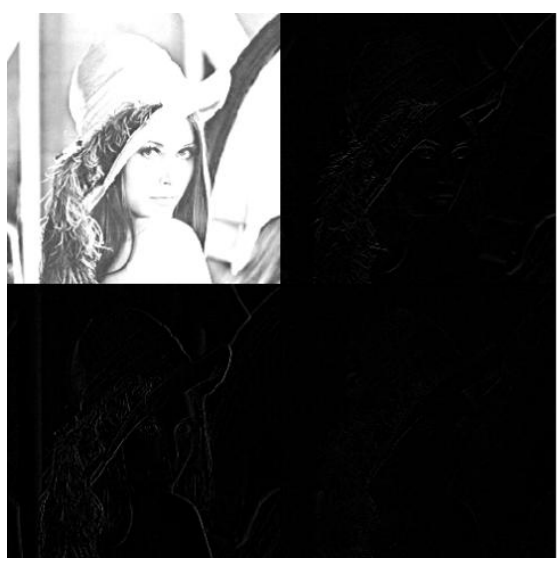

Figure 3: First level of decomposition 


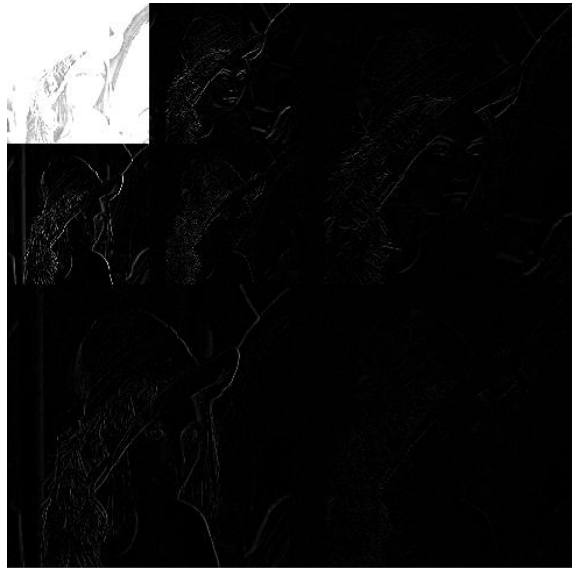

Figure 2: Second level of decomposition.

Median filter [5] works on medial pixel value of its surrounding neighbours. It preserves the smoothness in a resultant image. A median filter operates on pixel based noise reduction approach under structural constraints. In order to retain the smoothness and edges median filters are best choice among the other nonlinear filters.

In this paper median filtering and discrete Wavelet Transform are used for denoising corrupted images. Median filter of size 3X3, 5X5 and discrete Wavelet Transform are applied on the noisy images and resultant PSNR values are observed. Speckle, Salt \& Pepper and Gaussian noise are taken into consideration. Their individual and combined results have been observed.

\section{RESULTS}

This paper simulates the results for Gaussian, Speckle and Salt \& Pepper noise, for denoising median filter of size 3X3, $5 X 5$ and discrete wavelet Transform have been used. Then we have evaluated the results obtained on the basis of Peak signal to noise ratio. Graphs have been plotted by varying Noise density from 0.001 to 0.40 and PSNR corresponding to each value has been noted down

Figure 4 shows the noisy and reconstructed images with salt and pepper noise densities $0.01,0.05$ and 0.1 respectively. Figure 5 shows the noisy and reconstructed images with Gaussian noise densities $0.01,0.05$ and 0.1 respectively and Figure 6 shows the noisy and reconstructed images with speckle noise densities $0.01,0.05$ and 0.1 respectively.

Table 1 shows best improvement in the PSNR of the reconstructed image over the noisy image by applying combination of median filter and discrete wavelet Transform. Actually salt and pepper noise is removed most effectively by median filtering, but low density noise is removed well by median filter of $3 \mathrm{X} 3$ whereas $5 \mathrm{X} 5$ removes higher density noise. And DWT_med5 that is DWT combined with median filter of size $5 \mathrm{X} 5$ is best for removing Speckle and Gaussian noise.

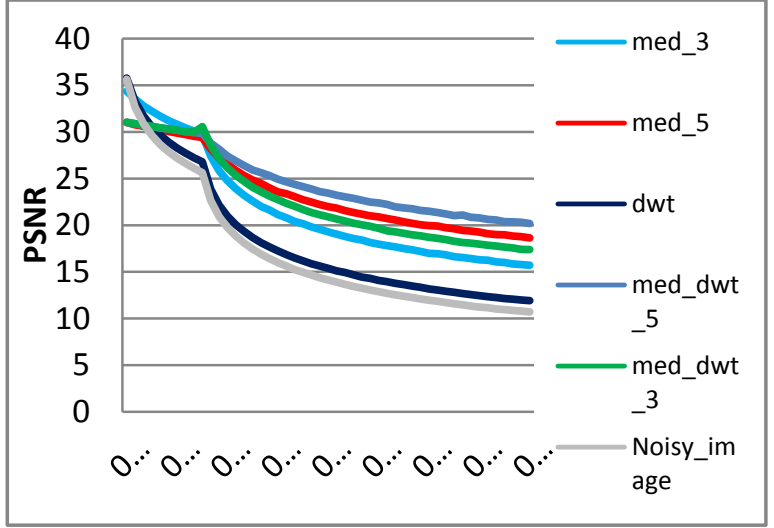

Figure 1: Speckle Noise Removal (PSNR vs Noise Density)

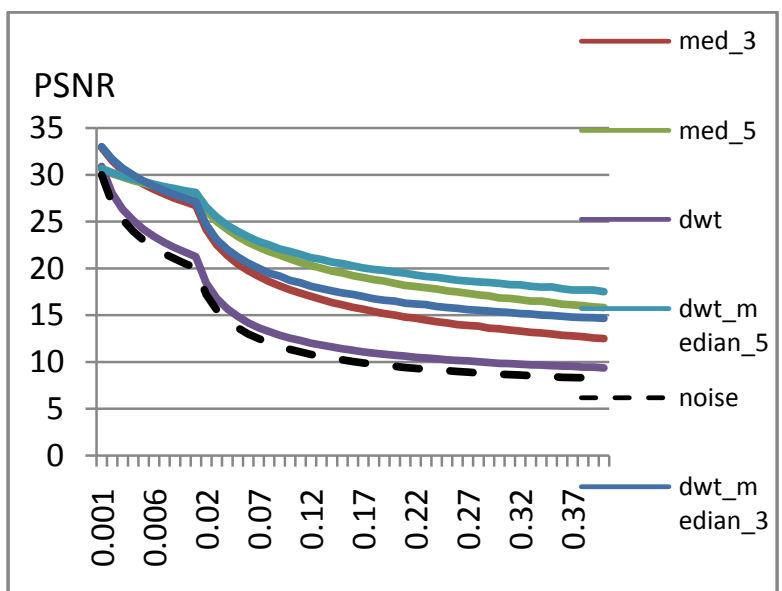

Figure 2: Gaussian Noise Removal (PSNR vs Noise Density)

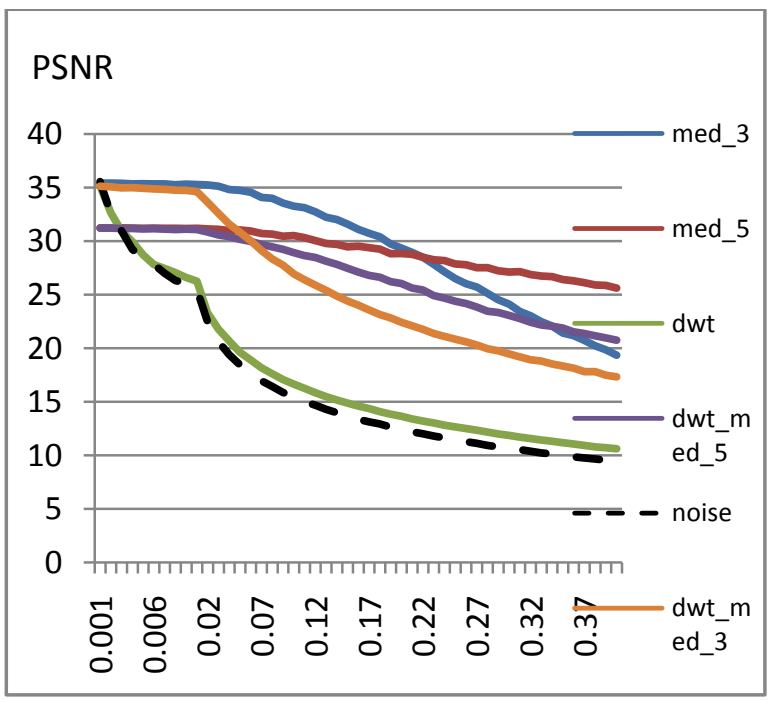

Figure 3: Salt \& Pepper Noise Removal (PSNR vs Noise Density) 
Table 1: Results of improved PSNR after denoising using median filtering and DWT

\begin{tabular}{|c|c|c|c|c|}
\hline S.No. & $\begin{array}{l}\text { Type of } \\
\text { Noise }\end{array}$ & $\begin{array}{c}\text { Noise } \\
\text { Density }\end{array}$ & $\begin{array}{l}\text { PSNR of } \\
\text { reconstruct } \\
\text { ed Image }\end{array}$ & $\begin{array}{c}\text { PSNR of } \\
\text { Noisy } \\
\text { Image }\end{array}$ \\
\hline \multirow[t]{7}{*}{1} & \multirow{4}{*}{$\begin{array}{l}\text { Salt and } \\
\text { Pepper } \\
(\operatorname{med} 3)\end{array}$} & 0.01 & 35.289 & 25.49 \\
\hline & & 0.1 & 33.26 & 15.48 \\
\hline & & 0.15 & 31.58 & 13.66 \\
\hline & & 0.2 & 29.35 & 12.48 \\
\hline & \multirow{3}{*}{$\begin{array}{l}\text { Salt and } \\
\text { Pepper } \\
\text { (med5) }\end{array}$} & 0.25 & 27.86 & 11.45 \\
\hline & & 0.30 & 27.122 & 11.45 \\
\hline & & 0.40 & 25.618 & 9.43 \\
\hline \multirow[t]{4}{*}{2} & \multirow{4}{*}{$\begin{array}{c}\text { Speckle } \\
\text { (DWT_m } \\
\text { ed5) }\end{array}$} & 0.01 & 29.844 & 25.6514 \\
\hline & & 0.05 & 26.889 & 18.8422 \\
\hline & & 0.1 & 24.929 & 16.005 \\
\hline & & 0.2 & 22.684 & 13.262 \\
\hline \multirow[t]{4}{*}{3} & \multirow{4}{*}{$\begin{array}{l}\text { Gaussian } \\
\text { (DWT_m } \\
\text { ed5) }\end{array}$} & 0.01 & 28.113 & 20.0361 \\
\hline & & 0.05 & 23.97 & 13.6436 \\
\hline & & 0.1 & 21.805 & 11.3307 \\
\hline & & 0.2 & 19.61 & 9.53 \\
\hline
\end{tabular}
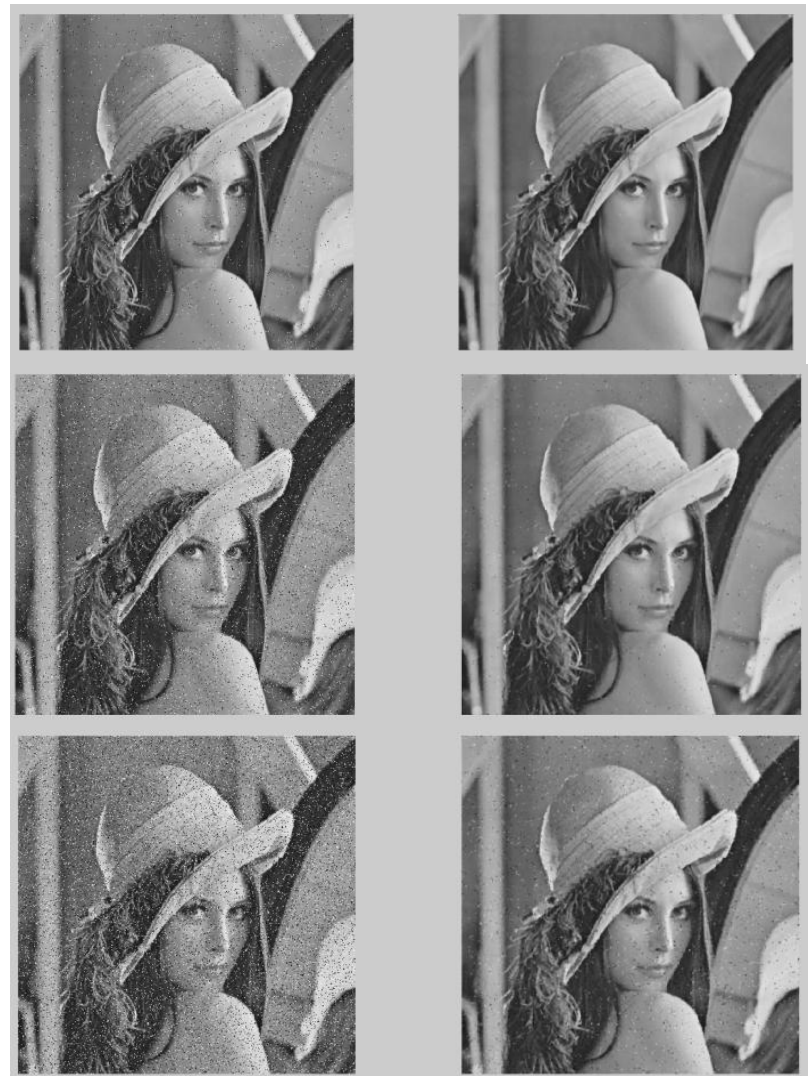

Figure 4: Noisy and reconstructed images with salt and pepper noise densities $0.01,0.05$ and 0.1 respectively
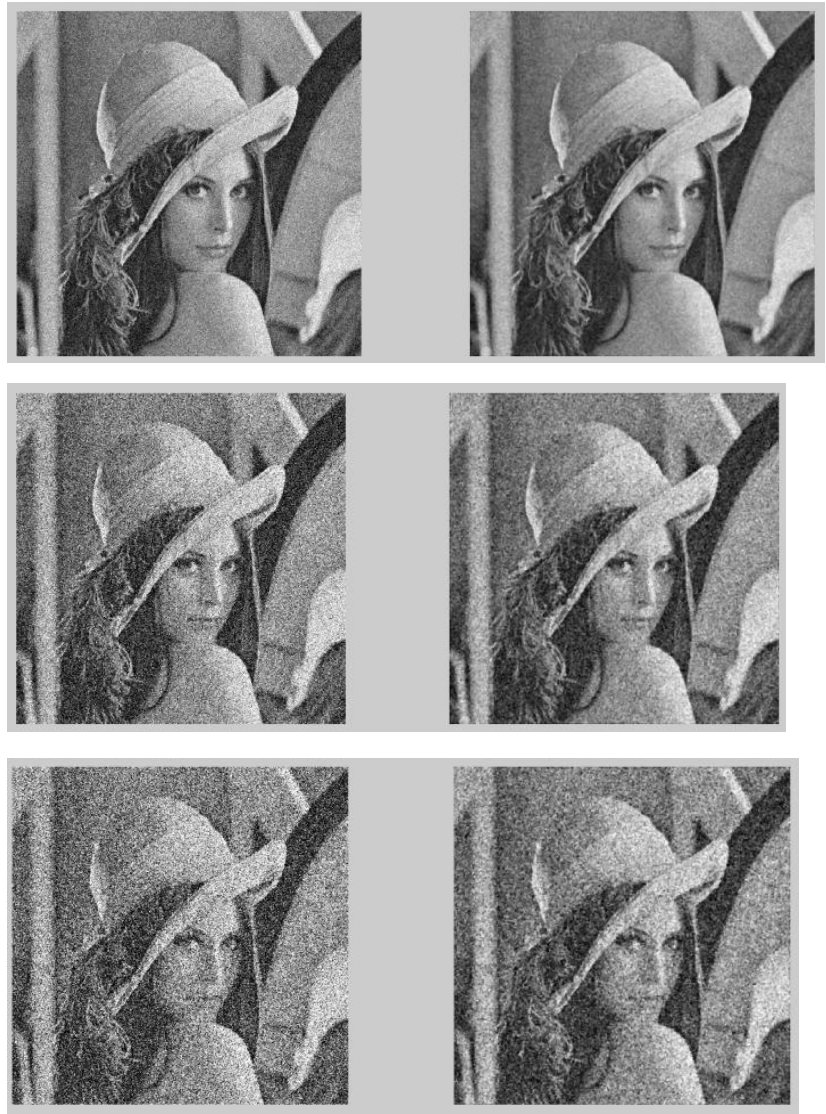

Figure 5: Noisy and reconstructed images with Gaussian noise densities $0.01,0.05$ and 0.1 respectively
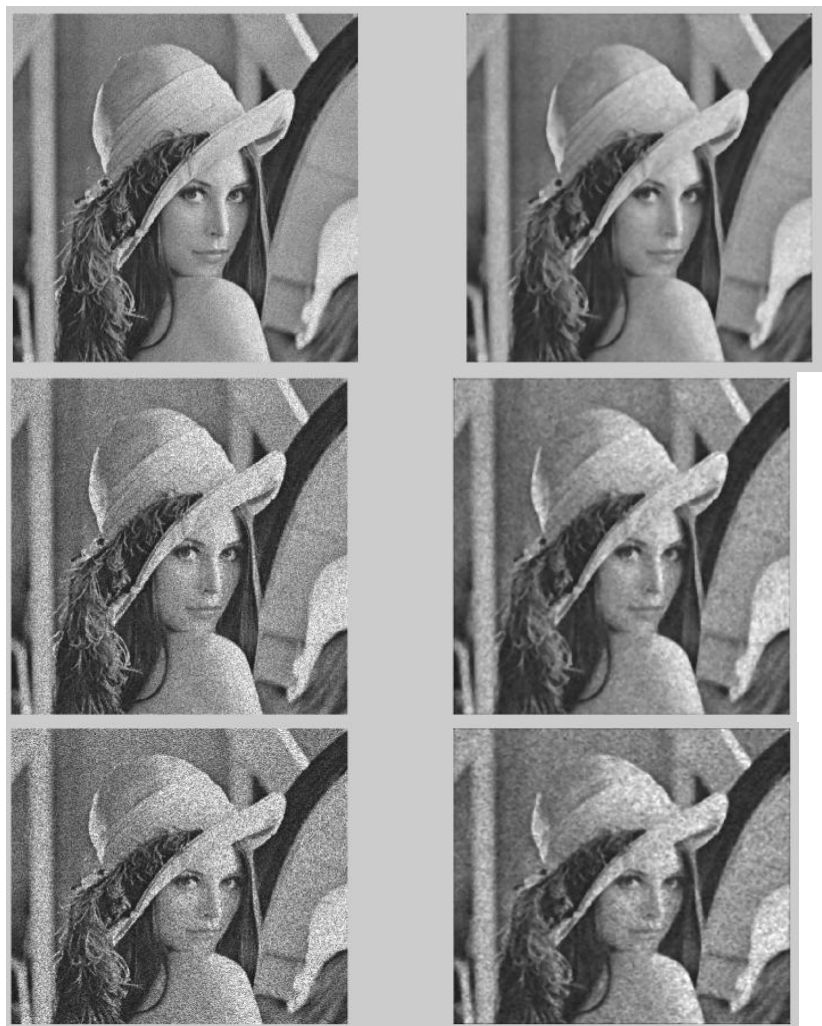

Figure 6: Noisy and reconstructed images with Speckle noise densities $0.01,0.05$ and 0.1 respectively 


\section{CONCLUSION}

In this paper denoising of noisy images have been performed using median filter of size $3 \times 3,5 \times 5$ and discrete wavelet Transform. The simulated results for Gaussian, Speckle and Salt \& Pepper noise are shown. PSNR has been used as evaluation criteria. By comparing the results obtained it can be concluded that Salt and pepper noise is removed most effectively by median filtering. Though low density noise is removed well by median filter of size $3 X 3,5 X 5$ size median filter is ideal for removing higher density noise. And DWT_med5 that is DWT combined with median filter of size $5 \mathrm{X} 5$ is best for removing Speckle and Gaussian noise.

\section{REFERENCES}

[1] Ajay Kumar Boyat and Brijendra Kumar Joshi,"A Review Paper: Noise Models in Digital Image Processing" Signal \& Image Processing : An International Journal (SIPIJ) Vol.6, No.2, April 2015

[2] Rinci Shrivastava, Ravi Mohan," Image Denoising Methods: A Survey" International Journal of Advanced Research in Computer and Communication Engineering Vol. 3, Issue 8, August 2014,pp 7808-7811

[3] Mukesh C. Motwani, Mukesh C. Gadiya, Rakhi C. Motwani, Frederick C. Harris, Jr, (2004) "Survey of Image Denoising Techniques," Proc. of GSPx 2004, Santa Clara Convention Center, Santa Clara, CA, pp. 2730.

[4] Boyat, A. and Joshi, B. K. (2013) "Image Denoising using Wavelet Transform and Median Filtering', IEEE
Nirma University International Conference on Engineering," Ahemdabad.

[5] Joshi, A., Boyat, A. and Joshi, B. K. (2014) "Impact of Wavelet Transform and Median Filtering on removal of Salt and Pepper noise in Digital Images," IEEE International Conference on Issues and Challenges in Intelligent Computing Techniques, Gaziabad.

[6] V. Gupta, R. Mahle ; R. S. Shriwas," Image denoising using wavelet transform method" Tenth International Conference on Wireless and Optical Communications Networks (WOCN), 2013

[7] Rafael C. Gonzalez, Richard E. Woods" Digital image processing" second edition, Prentice Hall

[8] Yali Liu," Image Denoising Method based on Threshold, Wavelet Transform and Genetic Algorithm" International Journal of Signal Processing, Image Processing and Pattern Recognition Vol. 8, No. 2 (2015), pp. $29-40$

[9] N.Kalyani , A.Velayudham," Analysis of Image Denoising Methods Using Various Wavelet Transform" International Journal of Advanced Research in Electronics and Communication Engineering (IJARECE) Volume 4, Issue 1, January 2015

[10] Lubna Gabralla , Hela Mahersia ,Marwan Zaroug," Denoising CT Images using wavelet transform" (IJACSA) International Journal of Advanced Computer Science and Applications, Vol. 6, No. 5, 2015 